\title{
GooGreen: Towards Increasing the Environmental Awareness of Households
}

\author{
Ruud Mattheij ${ }^{1}$, Lindsy Szilvasi ${ }^{1}$, Lorraine de Beer ${ }^{1}$, Kartini Rakiman ${ }^{1}$, \\ and Suleman Shahid ${ }^{2}$ \\ ${ }^{1}$ Department of Information and Communication Sciences Tilburg University, \\ Tilburg, the Netherlands \\ R.J.H.Mattheij@uvt.nl, L.M.F.Szilvasi@students.uu.nl, \\ L.M.A.T.deBeer@uvt.nl, K.C.Rakiman@uvt.nl \\ ${ }^{2}$ Tilburg centre for Cognition and Communication, \\ Tilburg University, Tilburg, the Netherlands \\ s.shahideuvt.nl
}

\begin{abstract}
In this paper, we present an interaction design exploration into the possibilities of using a computer application to increase the sustainability of households in an enjoyable manner. This is achieved by providing household members with personal information regarding their energy consumption and its related costs. This application also introduces a competition element that links the energy consumption in every household member's room to their personal user account in order to motivate users to compare their energy consumption with other household members. Early results show that the system indeed enhances the environmental awareness and that users are pleased with the idea of competition-based energy savings.
\end{abstract}

Keywords: Saving energy, sustainability, energy consumption, advice, control, competition element, environmental awareness.

\section{Introduction}

Sustainability and environmental awareness have been on the policy agenda for decades, resulting in many studies aiming to encourage households to reduce their energy consumption. Many of these studies showed that providing household members with information about ways to reduce their energy consumption tends to result in higher awareness, but does not necessarily change their behavior or actually aids them in saving energy [5]. Likewise, it is concluded that rewards can effectively encourage energy conservation [2], although mostly with short-lived effects [1].

Furthermore, related research indicates that improved feedback on energy consumption might provide households with a tool to control their energy consumption and, ultimately, improve their energy savings. It is also indicated that it is more effective to provide household members with electronic feedback of energy consumption than giving this information in hardcopy [8]. It is concluded that the most successful feedback combines the following features: long and frequently given feedback, the 
provision of an appliance-specific breakdown, presented clearly and in an appealing way and using computerized and interactive tools [3][4][6]. Based on these design principles, a number of applications have been designed for persuading households to control their energy consumption [7], although most of these applications only incorporate small set of design principles and miss many crucial ones, which play a key role in changing people's attitude towards energy consumption.

Therefore, it is hypothesized that an application that incorporates proven design principles and that allows households to view and control their energy consumption with a single application would provide household members with better insights concerning their energy consumption. It is also hypothesized that the introduction of a challenging competition element in the application might keep the application interesting to users for a longer period of time. It might also help in reducing boredom that rises over time. This would therefore increase the household's sustainability and may also lead to financial savings, thereby making the application financially attractive to households.

In this paper we present the GooGreen application, which aim is to test this hypothesis. The idea is to collect and combine all key design principles that are related to persuasive interfaces and sustainable technologies in one application and assist users in saving energy and maintaining a sustainable lifestyle in an enjoyable and extremely user-friendly manner.

\section{User Research}

From literature, it was clear that there is a need for an application that could help household members in saving energy. It was also apparent that changing the behavior of people towards energy consumption is a real challenge. This raised the question how household members use or waste energy and how they could be convinced to change their behavior. We therefore performed a proper user research, which consisted of observations and semi-structured interviews. We consulted literature and conducted a brainstorm session to prepare our questionnaire and questions.

These questions were combined into an interview, which was tested during a pilot test. Feedback from this test helped us to improve the interview. All questions in the interview were divided into four different categories/themes: environmental awareness (the degree and relevance of sustainability in the household), system content (preferred and/or required features of the application), system layout (the appearance/attractiveness of the application) and social activities (regarding a competition element of the application).

After a pre-pilot test, preliminary user studies were conducted. These user studies consisted of semi-structured interviews with 12 participants in their own familiar (home) environments: 7 men (average age 39) and 5 women (average age 41) using the interview and topics mentioned above.

From our user studies, we were not only able to confirm the previous findings [2][3][4], but we also found interesting information about Dutch households. A few of the important findings are as follows: 
- Participants are willing to increase their environmental awareness and sustainability, also if this would require them to use a computer application.

- They find it morally important to live sustainable, even if it requires changing some of their habits.

- Using such an application should not bring any major discomfort and should not be an extra overhead.

- This application should work as a supporting tool for enhancing environmental awareness.

- They should be able to use this application using a single device, at any time and at any place in the house, for controlling all appliances.

- Participants would like to be environmental friendly, although the most important reason for participants to use such an application will be to reduce costs and have a complete financial overview for the year.

- The real return on investment will be less expensive yearly bills. Many participants are worried about the increasing costs of energy and are annoyed by the extra amount of money they have to pay at the end of the year for extra energy consumption.

- A clear overview with proper feedback is extremely important for controlling the energy usage.

- Concerning the social activities, the main finding was that the majority of the participants would like to have a competition element in the application.

- The participants mentioned that it would be much more fun when they can compete with children and that children will find a fun element in this feature. They will also start contributing to a more sustainable lifestyle.

\section{Design Ideas}

The results of the user studies were discussed during a brainstorm session. The main results from this brainstorm session were that we had to narrow the scope of energy consumption to gas and electricity consumption. Likewise, the results indicated that we had to make the application very personal. Users should be provided with advice about their energy consumption, while they should also be able to control it. A competition element should be used in order to stimulate users to compare their energy consumption.

\section{Final Design}

The design ideas led to the development of a preliminary design of a paper-based low-fidelity prototype. The paper prototype was tested with 5 participants, which gave us interesting results about the contents and the visualization of the application. After the low-fi prototype, based on users' feedback, we decided that the application should contain clear schemes, symbols and icons, rather than text.

Likewise, we decided that the application should be touch-sensitive and that it should be possible to run it on a tablet pc. It was also decided that different layers of the house should be controllable; the entire house, a single floor or just a single room. The competition element was implemented by creating cartoon-like trees, which are connected to unique user accounts in the application. 


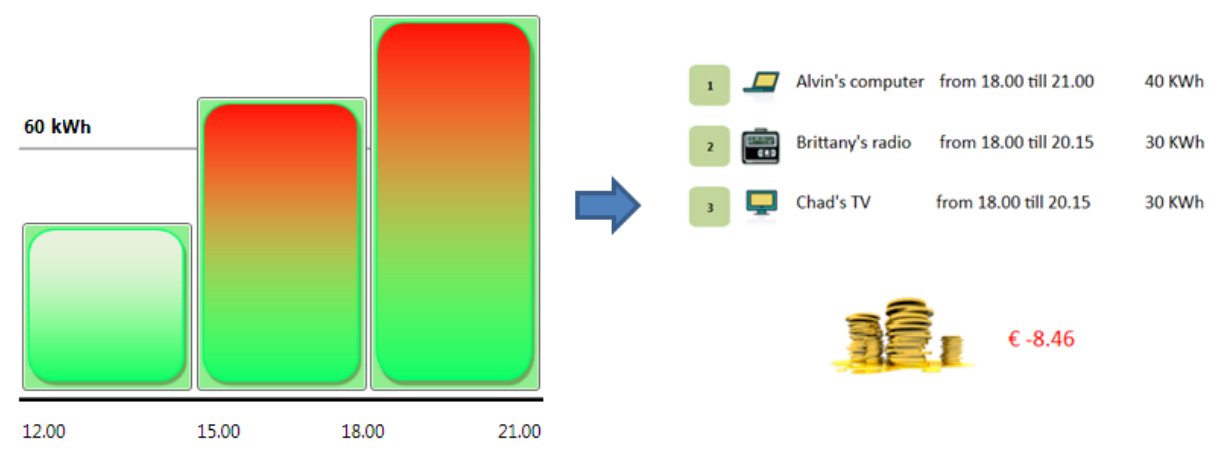

Fig. 1. The energy consumption on a particular period of time and the corresponding energy consuming devices

The final design contained three main function blocks: the Advice function, the View \& Control function and the Personalize function.

The Advice function informs users about the amount of energy that has been consumed by devices in the user's household during a certain period of time, using $\mathrm{m}^{3}$ for gas and kWh for electricity. "Gas" or "electricity" can be selected to consult all corresponding devices. The default backtrack period is 24 hours, but any length of time can be selected. Energy consumption is presented as a bar chart graph. A toolbar is available to select different periods of time, e.g. the last 24 hours or the last week.

More specific periods of time (e.g. from 12.00 to 15.00) can be consulted by selecting the corresponding bar in the bar chart graph to see which devices were powered on during this period of time. Finally, GooGreen calculates how much money the energy consumption has cost during this period of time. This informs users about devices that could be powered off more frequently in order to save energy. See fig. 1.

The View \& Control function informs users about the current power status (powered on or off) of devices. Devices can actually be powered on or off by this function. There are three different levels of control (see fig. 2), which are comparable with zooming in on a house:

Entire house; this is the top level of three recursive levels. GooGreen shows a simplified view of the house, displaying the number of gas and electricity consuming devices that are powered on at that moment on every floor of the house. This level of control should allow users to quickly power off all gas and/or electricity consuming devices in the house.

Single floor; on a floor level, this level of control is analog to the entire house level. There is a slight variation in the display of these levels, as pictured in fig. 2.

Single room; this level shows a list with devices and their power status (powered on or off) in that particular room. This level allows users to power specific devices on or off. Users select the gas icon in a room on the floor-level to navigate to a menu with an overview of all gas consuming devices in that particular room. 


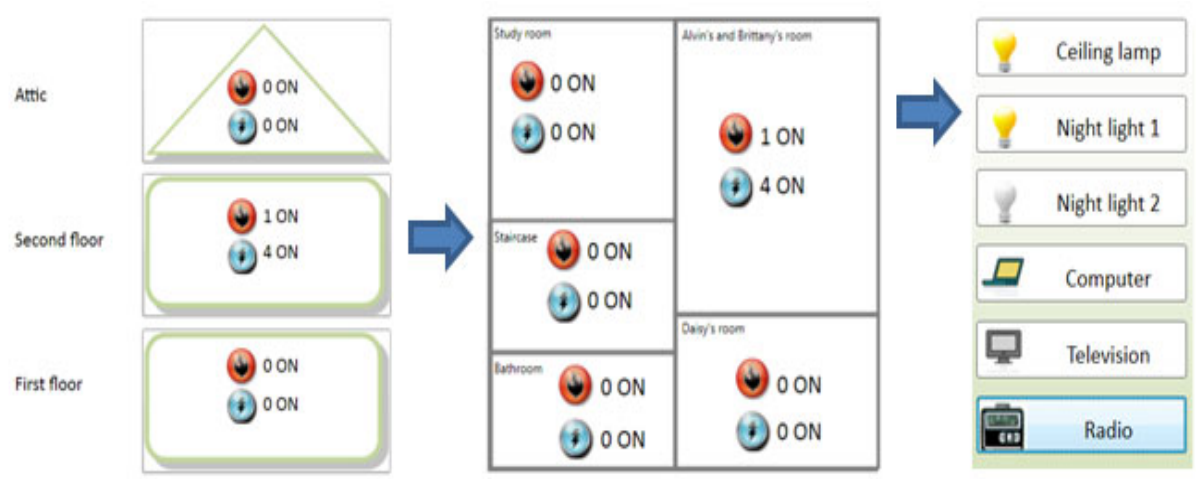

Fig. 2. The three level flow: house level (left), floor level (middle), room level (right)

In this overview, users can power these devices on or off. Likewise, selecting the electricity icon in a room on the floor-level navigates users to a menu with an overview of all electricity consuming devices in that particular room.

The Personalize function allows users to configure GooGreen according to their wishes. It is divided into two different kinds of settings: application settings (change GooGreen's appearance) and household settings (add, remove or change accounts). A virtual keyboard is available for text input. The GooGreen prototype uses an aura effect for highlighting interactive objects (e.g. a button) when users hover above them with their finger(s).

Every household member that uses GooGreen has a personal account. Every account has its own energy tree, a visual representation of the amount of energy that is consumed in the room of the account's owner. Higher energy consumption results in a less vital and unhealthy looking energy tree. Likewise, less energy consumption results in a more vital and healthy looking energy tree. See fig. 3.

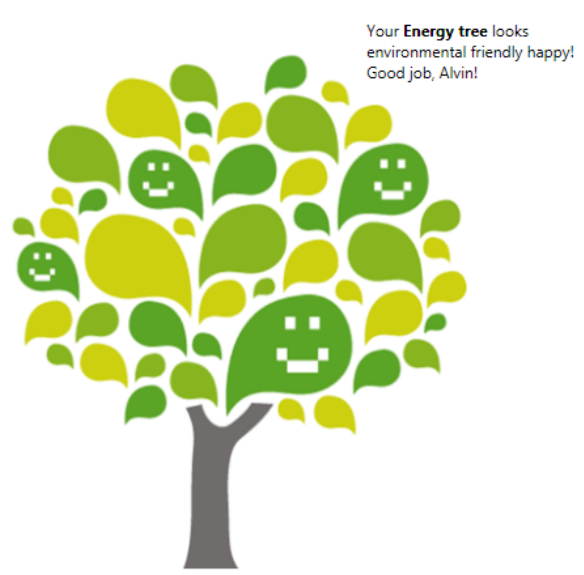

Fig. 3. The energy-tree provides an indication of the amount of energy a household member has used during a certain period of time. If a household member consumes more energy, the tree will look less healthy. 
The healthiness of the users' energy trees can be mutually compared to find out which user consumed least or most energy. See fig. 4.

\section{Implementation}

A high-fidelity prototype of GooGreen was developed using Visual Studio 2008. GooGreen uses the Windows Presentation Foundation (WPF) for its frontend and C\# for its backend. The high-fidelity prototype runs on Microsoft Windows tablet PCs. It requires the .NET 3.5 framework; a free software framework from Windows that enabled us to create the different graphic effects of GooGreen.

The information shown in GooGreen screens does not represent an actual house; the household, its members and its energy consumption are entirely fictional in order to prevent privacy related issues. This application requires devices that can be powered on or off remotely and that are easily accessible. These devices should be connected to the gas or electricity consuming household appliances that should be controlled by GooGreen.

\section{Evaluation}

The evaluation consisted of the heuristics evaluation and the usability tests. For the heuristics evaluation, we used the ten heuristic principles of Nielsen. Three expert reviewers tested the application for 10-15 minutes each. The usability test provided an indication of the quality of our solution by testing the time the participants needed to complete a task and the way they thought about GooGreen, thereby providing both quantitative and qualitative data. In the first phase of the usability evaluation, we wanted to measure the usability and acceptance of GooGreen. In the second phase, we would like to see its long-term impact on users' behaviors. The first evaluation consisted of a heuristics evaluation and a usability test.

Six people, 4 men (avg. age 31) and 2 women (avg. age 27) participated in our study. The test consisted of a pre and post-questionnaire and a task book with 11 key tasks.
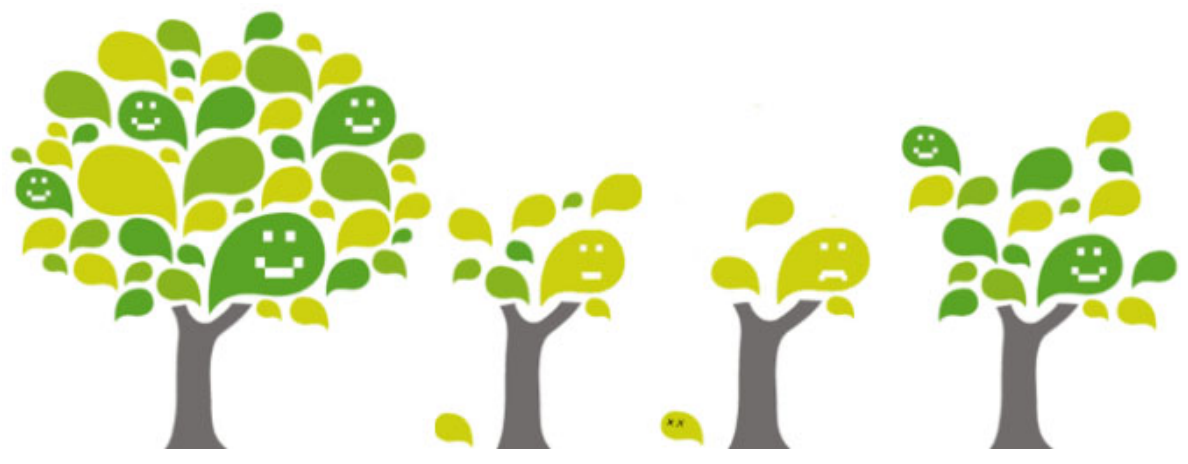

Fig. 4. The energy-trees can be mutually compared with other household members, thereby creating a competitive element. This specific example shows the energy trees of four different users with different states of 'environmental friendliness'. 


\section{Results and Discussion}

The mood test measured the participants' mood prior to the test by using a 1 to 7 Likert-scale; 1 means 'feeling very bad' and 7 means 'feeling very good'. The prequestionnaire was used to ask general questions concerning the users' overall experience with computers and their affinity with sustainability. The results indicated that participants had a reasonable affinity with computers, used computers for work, study and leisure time and that all participants were positively interested in sustainability.

The assignment consisted of 11 tasks. The time necessary for each task was measured and then compared with baseline time, which was set by the help of experts during the heuristic evaluation. The time necessary for each task was measured and compared with baseline time. Overall, all participants were able to finish their tasks in a reasonable amount of time. There were no unnecessary delays. Expertise in computer usage had a positive impact on completing the tasks, although this was not significant.

The first task consisted of starting up GooGreen, after which the participants were asked to retrieve information about the period in which they consumed most energy. All participants indicated that these tasks were easy to perform; it took most participants less than a minute to understand the advice function. After retrieving this information, all participants were asked to actually influence the energy consumption by performing a variety of scenarios, based on switching certain electric and/or gas consuming devices on or off. It took most participants about two minutes to understand this control function, which was mainly caused by the number of levels in the house (entire house, entire floor and entire room) the participants had to search in order to perform their tasks. Personalizing GooGreen to the preferences of the individual participants was a minor problem for most users. It took some participants a few minutes longer than the baseline to add a new user to the application. Consulting the high scores screen in order to compare the energy tree of the participants to other household members' energy trees was very convenient; all participants performed this task without problems.

After these experiments, a post-questionnaire was used to measure the attitude of participants towards GooGreen by asking users to what extent they agreed with the given statements. Their level of agreement was again measured on a 1 to 7 Likertscale; 1 means 'totally disagree' and 7 means 'totally agree'. Table 1 indicates that some tasks are apparently missing in the prototype. The participants' feedback was that they would appreciate "water consumption" to be included. Secondly, there was also a strong demand to include a feature to power on devices at a pre-programmed time.

It is also clear that the ratings for the first two questions are relatively low. This is due to the fact that the application was not fully functional as it was not directly attached to the physical devices. This had a little impact on the user experience and evaluation. Although users did not show great willingness to buy the product immediately, they totally understood the concept and most of them realized that GooGreen's interface is the main thing. 
Table 1. The statements that were served during the post-questionnaire and the corresponding level of agreement of the users

\begin{tabular}{lc}
\hline Statement & Average score \\
\hline $\begin{array}{l}\text { Now that I have tested GooGreen, I would pur- } \\
\text { chase it myself. }\end{array}$ & 4.2 \\
$\begin{array}{l}\text { Now that I have tested the application, I would } \\
\text { recommend it to others. }\end{array}$ & 4.8 \\
The application looks well-organized. & 6 \\
The application looks appealing. & 5.8 \\
The application is easy to use. & 5.6 \\
I can perform all tasks I would like to perform with & 1.6 \\
this application. & 6 \\
I think it is a good idea to implement the energy & \\
tree in the application as a competition element. & 6.2 \\
The energy tree is a fun part of the application & 6.2 \\
The energy tree looks appealing &
\end{tabular}

When compared to the mood test prior to the experiments, the results indicated that most users felt more satisfied and happier after using the GooGreen. They also thought that it is morally good to save energy and this feeling had a positive impact on their mood. According to the average scores, the application appeared to be appealing, well organized, easy to control and easy to use. Participants did not feel the application to be confusing. The experience with the energy tree was appreciated because of its appealing looks and the fun part of its competition element. The competition element turned out to be a very important feature and all participants unanimously appreciated it. It improved the application and was a motivating feature. Most participants considered it to be an effective way to stimulate the long-term use of the application. We learned from the user tests that this element should not be present at an individual level, but at a house level, community level or town level and that it may be spread among friends, especially in the case of children. They thought that this element is really motivating. On the other hand, they also showed a strong interest in privacy and the security of the system. They really wanted to have a controlled application, in which they decide 'who can see what'.

The results confirm the hypothesis that an application that allows households to control their energy consumption from one central point at home would provide household members with better insights concerning their energy consumption. Our project seems to support Fischer's conclusion as stated in the introduction.

Because of time constraints, the long term effects of the use of GooGreen could not be studied, although it is felt that this is an essential topic for a complete assessment of GooGreen. The data from the user tests is limited because the usability tests were performed with only few participants. Therefore, it is possible that results from usability tests with larger groups differ from our results. In any case, the results, especially the combination of the heuristics evaluation and usability test, do show a strong trend that not only confirm existing findings, but also give new insights about energy consumption and saving. 
GooGreen differs from other attempts to increase the sustainability of households because it contains a competition element (the energy tree concept and account system) and it allows users to influence energy consumption in their household in a systematic manner with minimal interaction. This solution also offers clear information about the costs of the energy consumption and it can work as an additional tool to manage its financial aspects. This approach makes consuming and saving energy very personal. It challenges users to become more involved with the sustainability of energy and resources.

This study also revealed that changing behavior is a long-term process and that this can only be achieved by a number of short-term milestones and rewards. Financial benefit is a major reward and an immediate feedback, which continuously updates users about their financial savings. Therefore, this will be the first real step towards a sustainable lifestyle.

\section{Conclusion and Future Work}

In this paper, we presented an interaction design exploration into the possibilities of using a computer application to increase the sustainability of households in an enjoyable manner. For this purpose, we developed the GooGreen application, which was designed in an iterative manner. Users did not only participate in the design of the application, but also evaluated its working version. Participants stated that GooGreen's presentation of information and advice regarding energy consumption increased their awareness about energy consumption considerably. Participants liked the idea to view and control their energy consumption in the household with a single application. The participants acknowledged the value of the competition element (the energy tree concept and account system) as a solution to involve more people in saving energy in a household. The initial use of this application was appreciated by most users.

This application, however, is still limited in a number of ways as it was started as a student project during a course. Therefore, the time period for development and evaluation was a little short. We could not build a fully functional system, which actually connects with physical devices. Likewise, we did not allow users to try this application in their daily routine on long-term basis.

For future research, we would like to extend this application's development and evaluation in a number of ways. First of all, we recommend a longitudinal investigation to determine the attractiveness of this solution. We also want to perform a costbenefit analysis to study the relationship between buying this application and the amount of money that can be saved by the help this application. Another important improvement is to develop an interface between this application and physical devices.

\section{References}

1. Abrahamse, W., Steg, L., Vlek, C., Rothengatter: A review of intervention studies aimed at Household energy conservation. Journal of Environmental Psychology 25(3), 273-291 (2005)

2. Bang, M., Torstensson, C., Katzeff, C.: The PowerHhouse: A Persuasive Computer Game Designed to Raise Awareness of Domestic Energy Consumption. In: IJsselsteijn, W.A., de Kort, Y.A.W., Midden, C., Eggen, B., van den Hoven, E. (eds.) PERSUASIVE 2006. LNCS, vol. 3962, pp. 123-132. Springer, Heidelberg (2006) 
3. Fischer, C.: Feedback on household electricity consumption: a tool for saving energy? Energy Efficiency 1(1), 79-104 (2008)

4. Fogg, B.J.: Persuasive technology: using computers to change what we think and do. Ubiquity 12, 89-120 (2002)

5. Heijs, W.J.M.: Household energy consumption: Habitual behavior and technology, pp. 149-157 (2006)

6. IJsselsteijn, W.A., de Kort, Y.A.W., Midden, C., Eggen, B., van den Hoven, E.: Persuasive Technology for Human Well-Being: Setting the Scene. In: IJsselsteijn, W.A., de Kort, Y.A.W., Midden, C., Eggen, B., van den Hoven, E. (eds.) PERSUASIVE 2006. LNCS, vol. 3962, pp. 1-5. Springer, Heidelberg (2006)

7. Kim, T., Hong, H., Magerko, B.: Designing for Persuasion: Toward Ambient EcoVisualization for Awareness. In: Ploug, T., Hasle, P., Oinas-Kukkonen, H. (eds.) PERSUASIVE 2010. LNCS, vol. 6137, pp. 106-116. Springer, Heidelberg (2010)

8. Wood, G., Newborough, M.: Dynamic energy-consumption indicators for domestic appliances: environment, behavior and design. Energy and Buildings 35(8), 821-841 (2003) 